\title{
Chest radiographs as predictors of length of stay in right-sided infective endocarditis
}

\author{
Grerk Sutamtewagul MD, Charoen Mankongpaisarnrung MD, \\ Nat Dumrongmongcolgul MD, Teerapat Nantsupawat MD, Kenneth Nugent MD
}

\begin{abstract}
Objective: Right-sided infective endocarditis (IE) occurs less frequently than left-sided IE and is usually caused by intravenous drug use or intravascular device-related infection. Septic pulmonary embolism can cause an abnormal chest radiograph (CXR), possibly raise pulmonary artery pressures, and may influence clinical and hospital outcomes.

Methods: We conducted a retrospective chart review of patients diagnosed with rightsided IE from January 2000 to December 2011. Clinical parameters were collected and analyzed to define patients' characteristics and their association with clinical outcomes, including length of stay (LOS).

Results: 208 eligible patients had a diagnosis of IE; 19 (9.1\%) had right-sided IE. Most were related to intravenous drug use (73.7\%) and presented with dyspnea and fever (55.6\%). $78.9 \%$ of patients had abnormal CXR (parenchymal involvement, cardiomegaly, or pleural effusion). Echocardiography revealed tricuspid valve involvement in all patients; the median mean pulmonary artery pressure (MPAP) by transthoracic echocardiographic estimation was $26.4 \mathrm{~mm} \mathrm{Hg}$. Patients with abnormal CXR had significantly longer LOS than those with normal CXR (21.4 vs. 7.5 days, $p=0.008)$. MPAP was not associated with LOS ( $p=0.72$ ).

Conclusions: Right-sided IE is often associated with intravenous drug use. The majority of these patients have mild pulmonary hypertension, which could be due to hyperdynamic circulation and probable septic emboli. CXR with pulmonary involvement may be useful in predicting the length of hospital stay in these patients and identifying patients with more complications.
\end{abstract}

Key words: endocarditis, chest radiographs, outcomes, length of stay

Corresponding author: Grerk Sutamtewagul, MD Contact Information: grerk.sutamtewagul@ ttuhsc.edu

DOI: 10.12746/swrccc2014.0206.066

\section{INTRODUCTION}

Right-sided infective endocarditis (IE) comprises $10 \%$ of IE cases and is caused by Staphylococcus aureus infections in $70 \%$ of the cases. ${ }^{1,2}$ It is usually a consequence of intravenous drug use (IVDU) and 
involves the tricuspid valve rather than the pulmonic valve. ${ }^{3-5}$ Patients with right-sided IE can have septic emboli in the lung and develop septicemic pneumonia, pulmonary infarcts, lung abscesses, bilateral pneumothoraces, and empyema. ${ }^{1,5,6}$ The clinical presentations, chest radiograph (CXR), and/or echocardiographic parameters may predict and /or influence the clinical outcomes in these patients. In particular, the size of vegetations influences prognosis. ${ }^{7}$ We speculated that embolic events and vascular changes associated with vegetations would increase pulmonary artery pressures, which, in turn, would predict hospital outcomes.

\section{Methods}

We conducted a retrospective review of the medical records of patients diagnosed with infective endocarditis between 2000 and 2011identified by ICD-9 code of 421.1. Only patients with right-sided IE were included in the study. Demographic and clinical data were collected and analyzed. Radiographic reports and images have been reviewed by the authors. Pulmonary artery systolic pressure (PASP) was tabulated from echocardiographic reports and used to calculate to mean pulmonary artery pressure (MPAP) according to equation: MPAP $=0.61 \times$ PASP +2 . Clinical outcomes included in-hospital complications (heart failure, septic or cardiogenic shock, pulmonary infarction, and acute kidney injury), length of stay, and mortality. Statistical analysis was performed using ttests, analysis of variance (ANOVA), chi-square tests, Pearson's correlation and non-parametric correlation, and Mann-Whitney $U$ tests using IBM SPSS Statistics version 20.0 (Armonk, NY). P-values $<0.05$ were considered statistically significant. This study was approved by the Institutional Review Board at Texas Tech University Health Sciences Center, Lubbock, Texas.

\section{RESULTS}

Echocardiography reports were reviewed from 208 patients with a discharge diagnosis of IE. Nineteen patients $(9.1 \%)$ had right-sided IE, $42 \%$ were men with a median age of 39 years (range 19-58), and the majority of the patients $(72.2 \%)$ were Caucasian (Table). Most patients had a history of IVDU $(n=$ $14,73.7 \%)$; two patients $(10.5 \%)$ had history of prosthetic valve replacement. These patients presented with dyspnea (55.6\%), fever (55.6\%), cough (27.8\%), and chest pain $(22.2 \%)$.

Fifteen patients (78.9\%) had abnormal CXR, including parenchymal involvement $(86.7 \%)$, cardiomegaly $(26.7 \%)$, pleural effusion $(26.7 \%)$, pulmonary congestion (13.3\%), and an enlarged pulmonary trunk $(6.7 \%)$. Echocardiographic studies revealed that all patients had tricuspid valve involvement, and 29\%, $47 \%$, and $12 \%$ were graded as having mild, moderate, and severe tricuspid regurgitation, respectively. Most of the vegetations $(62.5 \%)$ were moderate size with the greatest diameters between 5-10 mm. Pulmonary artery systolic pressure (PASP) from echocardiography ranged from $21-71 \mathrm{~mm} \mathrm{Hg}$ which translated into a mean pulmonary arterial pressure (MPAP) range of $14-45 \mathrm{~mm} \mathrm{Hg}$ (median MPAP $=26.4 \mathrm{~mm} \mathrm{Hg}$, interquartile range 25\%-75\%: $24-27 \mathrm{mmHg}$ ). Seven patients had positive blood cultures with Staphylococcus aureus bacteremia (58.3\%, of 12 patients with cultures), two had positive culture for $S$. epidermidis, and one had positive culture for viridans Streptococcus.

Complications from right-sided IE in this study included left-sided heart failure $(27.8 \%)$, hypotension $(21.1 \%)$, acute kidney injury $(21.1 \%$, defined by $50 \%$ increase of creatinine level), pulmonary abscess $(16.7 \%)$, right-sided heart failure $(10.5 \%)$, and pulmonary infarction (5.6\%). The median length of stay was 12 days (interquartile range 25\%-75\%: 9-28 days); there was one death. Surgical treatment was required in six patients (31.6\%); five patients had bioprosthetic valve replacement.

There was no correlation between PASP and length of stay $(r=-0.11$, $p$-value $=0.72)$. Patients with abnormal CXRs had longer lengths of stay than those with normal CXRs (mean length of stay \pm standard deviation $=21.4 \pm 15.3$ days vs. $7.5 \pm 2.4$ days, $p$ $=0.008)$. There was also no association between length of stay and age, ethnicity, body mass index, presence of comorbidities, or number of comorbidi- 
ties in this study. Mean PASP in patients with normal CXR and with parenchymal involvement group were $29.2 \pm 25.1 \mathrm{mmHg}$ and $25.8 \pm 4.2 \mathrm{mmHg}$, respectively $(p=0.73)$.
5-10 percent of all IE cases. ${ }^{8}$ Possible explanations include the lower rate of right-sided congenital heart disease, the lower pressure and shear stress on rightsided valvular structures, and the lower oxygen con-

\section{Table Patient demographics and characteristics}

\begin{tabular}{|c|c|c|}
\hline Patient Characteristics & Frequency & Percent \\
\hline \multicolumn{3}{|l|}{ Gender } \\
\hline Male & 8 & 42.1 \\
\hline Female & 11 & 57.9 \\
\hline \multicolumn{3}{|l|}{ Race } \\
\hline White & 13 & 72.2 \\
\hline Hispanic & 2 & 11.1 \\
\hline African-American & 2 & 11.1 \\
\hline Age (years) & Median 39 & Range 19-58 \\
\hline \multicolumn{3}{|l|}{ Comorbidity } \\
\hline Liver cirrhosis or viral hepatitis & 7 & 38.9 \\
\hline Hypertension & 5 & 26.3 \\
\hline Chronic kidney disease & 3 & 15.8 \\
\hline Alcoholism & 2 & 10.5 \\
\hline History of intravenous drug use & 14 & 73.7 \\
\hline History of prosthetic heart valve & 2 & 10.5 \\
\hline History of dental procedure & 0 & 0 \\
\hline Presence of intravascular device & 0 & 0 \\
\hline
\end{tabular}

\section{Discussion}

The incidence of right-sided IE is significantly lower than left-sided IE and usually ranges between tent of venous blood. However, among intravenous drug users, the proportion of right-sided IE increases to $86 \%$ and is even higher in those who have concurrent HIV infection with low CD4+ lymphocyte count. ${ }^{1,9}$ 
Even though IVDU is the classic cause of right-sided $\mathrm{IE}$, intravenous catheters and intracardiac devices are also important causes.

The relationship between IE and pulmonary hypertension has not been clearly elucidated. Our patients had mild pulmonary hypertension in the majority of cases. However, accurate MPAP measurements from right-sided heart catheterization were not performed, resulting in a limited analysis between actual MPAP and clinical parameters in our study. Hyperdynamic circulation from sepsis, pulmonary septic emboli, pulmonary granulomatosis from injection of "stabilizer" from drug abuse, and HIV infection can cause pulmonary hypertension. In our series, however, HIV infection is not a factor since only one patient was HIV-positive. Pulmonary septic emboli and infiltrates seen on the chest radiographs probably do not explain pulmonary hypertension either since there was no significant difference in MPAP between the normal chest radiograph and abnormal CXR (parenchymal involvement) groups. Although there was no definite evidence, subclinical septic embolism to the lungs could be a plausible cause of pulmonary hypertension in these patients.

Parenchymal involvement was common in our series and was also associated with increased length of stay. This reflected the importance of pulmonary involvement in right-sided IE. The pathology of parenchymal involvement is likely complex and includes septic embolism, parenchymal infarction, and community-acquired and health-care associated pneumonia. These possibilities would increase the length of hospital stay.

The role of septic pulmonary embolism in pulmonary hypertension is uncertain. Studies of thrombotic pulmonary embolism revealed that pulmonary embolism must occlude more than $30 \%$ of pulmonary vasculature to cause pulmonary hypertension. ${ }^{10}$ In addition to mechanical acute obstruction, there are also changes in the small vessels downstream from the emboli including medial hypertrophy, fibrointimal hyperplasia, microvascular thrombosis, and plexiform lesion. ${ }^{11}$ Thrombus formation and thrombolysis also release pro-inflammatory cytokines and vasoactive factors contributing to those changes. ${ }^{12}$ Septic pulmonary embolism can also cause mechanical obstruction and elicits inflammation. The mechanisms of development of pulmonary hypertension are probably similar to those of chronic thromboembolism with possibly more inflammation.

The limitations of our findings include the retrospective, single-center study design, small number of patients in the analysis, and incomplete or missing data. Moreover, none of the patients included had right heart catheterization done. Abnormality on the CXR may indicate other concomitant acute or chronic disease processes, which may not directly related to right-sided IE.

\section{Conclusion}

Right-sided IE often occurs in Staphylococcal septicemia and is usually associated with intravenous drug use. The majority of these patients have pulmonary hypertension, possibly from septic emboli. However, there was no association between PASP and length of stay. CXR can help predict the length of hospital stay in these patients. Additional studies with more patients and better statistical power are needed to provide more information on the importance of echocardiographic parameters on the clinical course in patients with right-sided IE.

KEYwords- endocarditis, chest radiographs, outcomes, length of stay

\footnotetext{
Received: $12 / 23 / 2014$

Accepted: 4/2/2014

Reviewers: Cihan Cevik MD

Published electronically: 4/15/2014

Conflict of Interest Disclosures: None
}

Author Affiliation: Drs Grerk Sutamtewagul, Charoen Mankongpaisarnrung, Nat Dumrongmongcolgul, and Teerapat Nantsupawat are residents in the Department of Internal Medicine at TTUHSC. Kenneth Nugent is a pulmonary physician in the Department of Internal Medicine. 


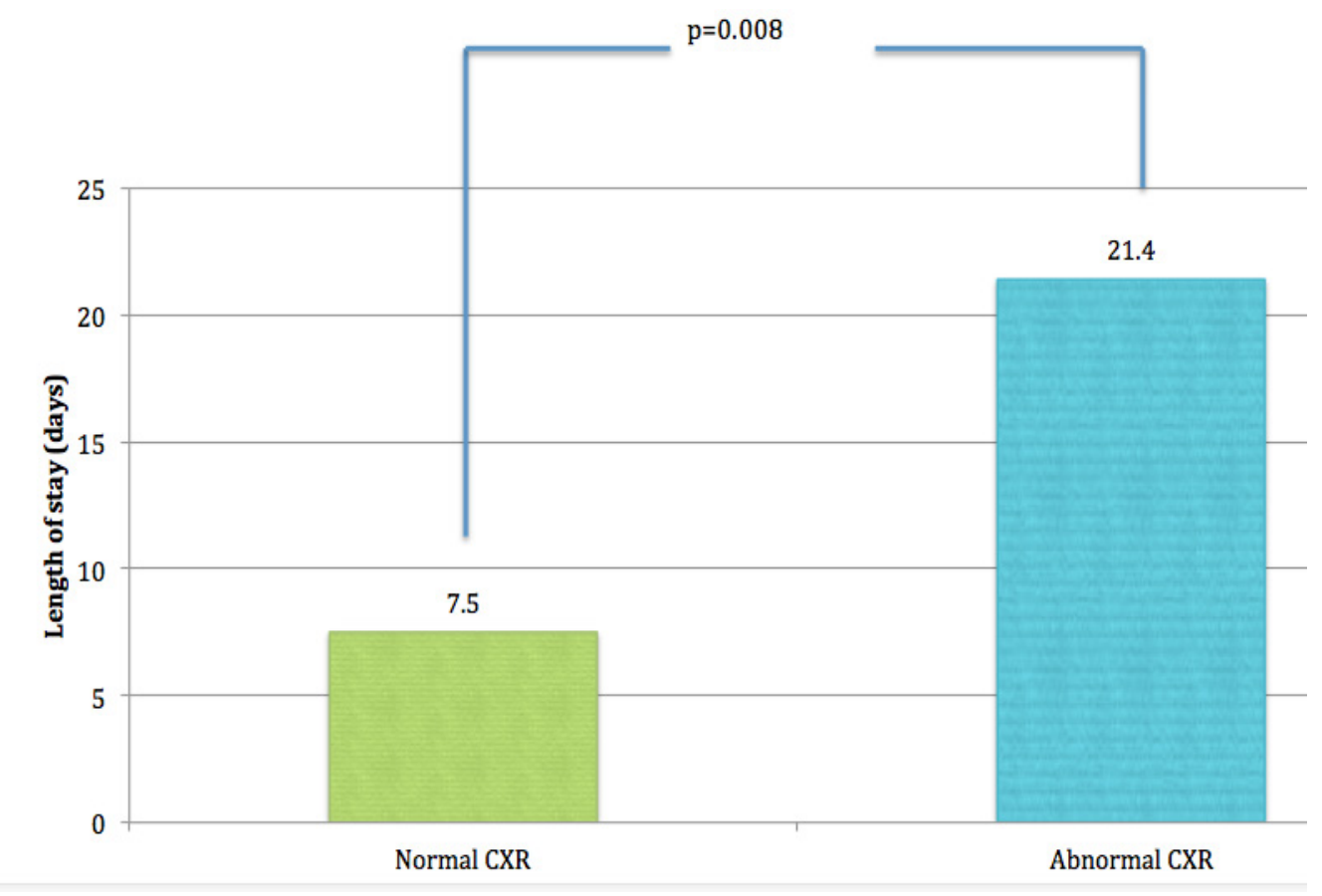

\section{REFERENCES}

1. Moss R, Munt B. Injection drug use and right sided endocarditis. Heart 2003;89:577-81.

2. Revilla A, Lopez J, Villacorta E, et al. Isolated right-sided valvular endocarditis in non-intravenous drug users. Revista espanola de cardiologia 2008;61:1253-9.

3. Jain V, Yang MH, Kovacicova-Lezcano G, Juhle LS, Bolger AF, Winston LG. Infective endocarditis in an urban medical center: association of individual drugs with valvular involvement. The Journal of infection 2008;57:132-8.

4. Jolobe OM. Right-sided endocarditis in the nondrug addict. Postgraduate Medical J 1994;70:54.

5. Mathura KC, Thapa N, Rauniyar A, Magar A, Gurubacharya DL, Karki DB. Injection drug use and tricuspid valve endocarditis. Kathmandu Univ Med J (KUMJ) 2005;3:84-6.

6. Nalos M, Huang SJ, Ting I, McLean AS. Diagnoses of right-sided empyema complicating tricuspid valve endocarditis during transesophageal echocardiography. Journal of the American Society of Echocardiography : official publication of the American Society of Echocardiography 2004;17:464-5.

7. Hecht SR, Berger M. Right-sided endocarditis in intravenous drug users. Prognostic features in 102 episodes. Annals of internal medicine 1992;117:560-6.

8. Chan P, Ogilby JD, Segal B. Tricuspid valve endocarditis. American heart journal 1989;117:1140-6.
9. Akinosoglou K, Apostolakis E, Marangos M, Pasvol G. Native valve right sided infective endocarditis. European journal of internal medicine 2013;24:510-9.

10. McIntyre KM, Sasahara AA. The hemodynamic response to pulmonary embolism in patients without prior cardiopulmonary disease. The American journal of cardiology 1971;28:288-94.

11. Klok FA, Mos IC, van Kralingen KW, Vahl JE, Huisman MV. Chronic pulmonary embolism and pulmonary hypertension. Seminars in respiratory and critical care medicine 2012;33:199-204.

12. Tuder RM, Abman SH, Braun T, et al. Development and pathology of pulmonary hypertension. Journal of the American College of Cardiology 2009;54:S3-9. 